\title{
COVID-19 and Sodium-Glucose Cotransporter 2 Inhibitors: No Fear to Attempt?
}

Novel coronavirus infectious disease (COVID-19) has been recognised as a pandemic by the World Health Organization (WHO) [1]. Mortality and morbidity are higher in elderly individuals and those with comorbidities, such as diabetes mellitus (DM), obesity, hypertension, respiratory tract diseases, coronary heart disease or cancer [1]. Indeed, two thirds of individuals who died from COVID-19 had DM in Italy [2].

Importantly, Chen et al. [3] have demonstrated that COVID-19 may exert a significant deleterious effect on myocardium and cardiac function. Moreover, some patients with COVID-19 develop acute kidney injury (AKI), which carries a worse prognosis [4].

Given the importance of DM in the outcomes of COVID-19 [5], antidiabetic treatment options and the need to achieve adequate glycaemic control are increasingly being discussed $[5,6]$. Of relevance, sodium-glucose cotransporter 2 inhibitors (SGLT2is) are known to reduce cardiovascular events and to protect kidney function [7] in type 2 DM. Thus, it appears worth examining the potential effect of SGLT-2is on preventing and/or delaying the development of cardiovascular events and $\mathrm{AKI}$ in patients hospitalised with acute COVID-19, regardless of diabetes.

In this setting, two trials with dapagliflozin as a cardioprotective and renoprotective agent in COVID-19 have already been initiated: DARE-19 (Dapagliflozin in respiratory failure in patients with COVID-19) [8] and TACTIC-E (Multi-Arm therapeutic study in pre-ICU patients admitted with Covid-19 experimental drugs and mechanisms) [9]. The rationale for these trials is that SGLT-2is not only improve heart failure and renal function in type 2 DM [7], but they also appear to reduce inflammation, hypoxia and excess cytokine release $[10,11]$. Importantly, the latter is known to play a pivotal role in adverse outcomes in COVID-19 [12,13]. In DARE-19 (900 participants), inclusion criteria are hospitalisation for mild-to-moder- ate COVID-19 (arterial oxygen saturation $\geq 94 \%$ with low-flow supplemental oxygen) of any duration and at least one of the following: hypertension; type 2 DM; atherosclerotic cardiovascular disease; heart failure; chronic kidney disease stage 3 to 4 [8]. Patients will be randomised (1:1) to dapagliflozin $10 \mathrm{mg}$ daily or placebo for 30 days and followed up for a total of 60 days [8]. The primary endpoint will be time to first death from any cause or new/aggravated organ dysfunction [8]. In TACTIC-E, 1407 patients with COVID-19 without need for intensive care unit (ICU) will be enrolled [9]. These will be randomised $(1: 1: 1)$ to either the experimental drug EDP1815 (preparation of a single strain of Prevotella histicola) or dapagliflozin $10 \mathrm{mg}$ daily plus ambrisentan (endothelin receptor antagonist) or standard care alone [9]. The primary endpoint will be time to the composite endpoint comprising death, mechanical ventilation, extracorporeal membrane oxygenation, cardiovascular support or renal failure [9]. In DARE-19 [8] and TACTIC-E [9], design and endpoints are based on the known renoprotective and cardioprotective properties of SGLT-2is [7, 11, 14] and on accepted meaningful parameters of COVID-19 clinical course [1-3].

Whether, indeed, SGLT-2is will prove beneficial in this new condition is currently a matter of investigation and hope. Potential helpful mechanisms include, among others, increased haematocrit, slight increase of ketone bodies, as well as reduction of endothelial dysfunction, glomerular hyperfiltration, oxidative stress and inflammation $[9,11,14]$. Certainly, caution is needed until we obtain tangible data on their utility or otherwise. However, unlike the Shakespearean hero, we should not lose potential benefits by "fearing to attempt" [15].

\section{Funding}

No funding or sponsorship was received for this study or publication of this article.

\author{
Conflict of Interest
}

NP has been an advisory board member of Astra-Zeneca, Boehringer Ingelheim, MSD, Novo Nordisk, Pfizer, Takeda and TrigoCare International; has participated in sponsored studies by Astra-Zeneca, Eli-Lilly, GSK, MSD, Novo Nordisk, Novartis and Sanofi-Aventis; has received honoraria as a speaker for Astra-Zeneca, Boehringer Ingelheim, Eli-Lilly, Elpen, MSD, Mylan, Novo Nordisk, Pfizer, Sanofi-Aventis and Vianex; and attended conferences sponsored by TrigoCare International, Eli-Lilly, Galenica, Novo Nordisk, Pfizer and Sanofi-Aventis. APS has given talks, attended conferences, and participated in advisory boards, and clinical trials sponsored by various pharmaceutical companies and she is currently Vice-President, National Diabetes Commission, Ministry of Health, Romania.

\section{References}

[1] Wang D, Hu B, Hu C et al. Clinical Characteristics of 138 hospitalized patients with 2019 novel coronavirus-infected pneumonia in Wuhan, China. JAMA 2020; 323: 1061-1069

[2] Remuzzi A, Remuzzi G. COVID-19 and Italy: what next? Lancet 2020; 395: 1225-1228

[3] Chen C, Chen C, Yan JT et al. Analysis of myocardial injury in patients with COVID-19 and association between concomitant cardiovascular diseases and severity of COVID-19. Zhonghua Xin Xue Guan Bing Za Zhi 2020; 48: E008

[4] Cheng Y, Luo R, Wang K et al. Kidney disease is associated with in-hospital death of patients with COVID-19. Kidney Int 2020; 97: 829-838

[5] Ceriello A. Hyperglycemia and the worse prognosis of COVID-19. Why a fast blood glucose control should be mandatory. Diabetes Res Clin Pract 2020; 163: 108186

[6] Penlioglou T, Papachristou S, Papanas N. COVID-19 and diabetes mellitus: May old anti-diabetic agents become the new philosopher's stone? Diabetes Ther 2020; 11: 1-3

[7] Zelniker TA, Wiviott SD, Raz I et al. SGLT2 inhibitors for primary and secondary prevention of cardiovascular and renal outcomes in type 2 diabetes: A systematic review and meta-analysis of cardiovascular outcome trials. Lancet 2019; 393: 31-39 
[8] US National Library of Medicine. Dapagliflozin in respiratory failure in patients with COVID-19; DARE-19. https://clinicaltrials.gov/ ct2/show/NCT04350593. Last accessed: 01 August 2020

[9] US National Library of Medicine. MulTi-Arm Therapeutic Study in Pre-ICu Patients Admitted With Covid-19 - Experimental Drugs and Mechanisms. https://clinicaltrials. gov/ct2/show/NCT04393246. Last accessed: 01 August 2020

[10] Packer M. Do sodium-glucose co-transporter 2 inhibitors prevent heart failure with a preserved ejection fraction by counterbalancing the effects of leptin? Diabetes Obes Metab 2018; 20: 1361-1366

[11] Tsioufis C, Andrikou E, Thomopoulos C et al. Oral glucose-lowering drugs and cardiovascular outcomes: from the negative RECORD and ACCORD to neutral TECOS and promising EMPA-REG. Curr Vasc Pharmacol 2017; 15: 457-468

[12] lannaccone G, Scacciavillani R, Del Buono MG et al. Weathering the cytokine storm in COVID-19: therapeutic implications. Cardiorenal Med 2020; June 29 [Epub ahead of print]

[13] Gonzalez-Rubio J, Navarro-Lopez C, Lopez-Najera E et al. Cytokine release syndrome (CRS) and nicotine in COVID-19 patients: Trying to calm the storm. Front Immunol 2020; 11: 1359
[14] Kawanami D, Matoba K, Takeda Y et al. SGLT2 inhibitors as a therapeutic option for diabetic nephropathy. Int J Mol Sci 2017; 18: 1083

[15] Shakespeare W. Measure for Measure. In: Wells S, Taylor G, Eds. The complete works of William Shakespeare. $1^{\text {st }}$ Edition. Oxford University Press; Oxford: 1988: 794

\section{Authors \\ Stella Papachristou ${ }^{1}$, Theano Penlioglou ${ }^{1}$ Anca Pantea Stoian², Nikolaos Papanas ${ }^{1}$}

\section{Affiliations}

1 Diabetes Centre, Second Department of Internal Medicine, Democritus University of Thrace, Alexandroupolis, Greece

2 Diabetes, Nutrition and Metabolic Diseases Department, "Carol Davila" University of Medicine, Bucharest, Romania

\section{Key words}

diabetes, autoimmunity, COVID-19, SGLT-2

inhibitors, treatment received 26.05.2020

revised $\quad 02.08 .2020$

accepted $\quad 06.08 .2020$

published online $\quad 10.09 .2020$

\section{Correspondence}

Prof. Nikolaos Papanas

Diabetes Centre

Second Department of Internal Medicine Democritus University of Thrace

Alexandroupolis

Greece

Tel.: + 3025513 51723, Fax: + 302551351723

papanasnikos@yahoo.gr

Bibliography

Exp Clin Endocrinol Diabetes 2022; 130: 198-199

DOI 10.1055/a-1235-5617

ISSN $0947-7349$

(C) 2020. Thieme. All rights reserved. Georg Thieme Verlag KG Rüdigerstraße 14 70469 Stuttgart, Germany 